\title{
Habilidades y competencias generales de los profesionales en la cadena de suministro en Panamá Oeste
}

\section{Skills and general competencies of professionals in the supply chain in Western Panama}

\author{
José Juan Valdés ${ }^{1}$, Malihe Jamal $^{1 *}$, Manuela Sánchez ${ }^{1}$ \& Milena Gómez-Cedeño ${ }^{2}$ \\ ${ }^{1}$ Licenciatura en Ingeniería Industrial - Centro Regional de Panamá Oeste - Universidad Tecnológica de Panamá \\ ${ }^{2}$ Profesora Asesora-Facultad de Ingeniería Industrial-Universidad Tecnológica de Panamá
}

\begin{abstract}
Resumen Para que las empresas logren el éxito en la gestión de la cadena de suministro interna, es fundamental que sus colaboradores cuenten con las habilidades y competencias requeridas para ello. En esta dirección, se busca identificar cuáles son las habilidades y competencias de mayor importancia y desempeño para la SCM en Panamá Oeste. De esta manera, se ha encontrado un estudio donde se enfatizan las 48 habilidades y competencias requeridas para el éxito de las empresas en la cadena de suministro por Gómez-Cedeño (2017), en el cual procedimos a realizar una encuesta a los estudiantes de Logística e Ingeniería Industrial. Mediante el análisis de la Matriz del IPM se obtuvo las habilidades de mayor importancia para las empresas, pero de bajo desempeño de los estudiantes: Finanzas y Gestión Presupuestaria y la Habilidad de Tecnología de la Información. De esta manera, recomendamos reforzar estas habilidades a través de nuevas asignaturas, metodologías de la enseñanza innovadoras, prácticas laborales y talleres en empresas para mejorar el desempeño de los futuros profesionales.
\end{abstract}

Palabras clave Gestión de la cadena de suministro, habilidades y competencias, matriz (IPM).

Abstract In order for companies to achieve success in the management of the internal supply chain, it is essential that their employees have the skills and competencies required for this. In this direction, we seek to identify the most important skills and competencies for the SCM in West Panama. In this way, a study has been found that emphasizes the 48 skills and competencies required for the success of companies in the supply chain by Gómez-Cedeño (2017). Based on this study, we proceeded to conduct a survey of Logistics students and Industrial Engineering. Through the analysis of the IPM Matrix, the most important skills for companies were obtained, but with low student performance: Finance and Budget Management and Information Technology Skill. In this way, we recommend strengthening these skills through new subjects, innovative teaching methodologies, work practices and workshops in companies to improve the performance of future professionals.

Keywords Supply chain management, skills and competencies, matrix (IPM).

* Corresponding authors: malihe.0797@gmail.com

\section{INTRODUCCIÓN}

Las empresas enfrentan grandes retos en la gestión de la cadena de suministro, debido a la competencia en este mercado globalizado para lo cual han optado por mejorar la planificación de la cadena de suministro. Para ello se preocupan por el reclutamiento adecuado del personal capacitado, para obtener mayor confiabilidad y éxito.

Según, Fawcett, Magnan, \& McCarter (2008), la educación y el entrenamiento son puentes para mejorar la productividad en las organizaciones. A pesar de que existe poca investigación sobre las competencias esenciales en la Gestión de la cadena de suministro (SCM), es importante reforzar los lazos entre el campo de estudio de la cadena de suministro y gestión de recursos humanos (HRM) [1].

La Gestión de la cadena de suministro (SCM) es la integración de los procesos clave de negocio en el suministro de productos, servicios e información, que añaden valor, desde los proveedores hasta el usuario final. En este sentido, la SCM tiene una importante dimensión humana debido a su énfasis en la comunicación y la cooperación entre todas las partes que comprenden la cadena de suministro (SC) [2].

En este mundo laboral las empresas imponen más interés hacia los profesionales capacitados, con habilidades interpersonales, buena comunicación, liderazgo y buena ejecución frente al campo laboral. En base a lo anterior, el propósito de las universidades es capacitar a futuros 
profesionales que satisfagan los requerimientos de las empresas hoy en día [1].

El presente artículo pretende identificar las habilidades y competencias que deben poseer los estudiantes para satisfacer al éxito de la cadena de suministros de las empresas de Panamá Oeste. Para ello se compara la matriz IPM de las empresas, reconociendo las habilidades de mayor importancia para la SCM.

De esta manera, procedimos a realizar encuestas a los estudiantes de Logística e Ingeniería Industrial que cursan sus últimos semestres en la Universidad Tecnológica de Panamá Centro Regional Panamá Oeste y empresas de producción con cadena de suministro en Panamá Oeste. Al obtener los resultados, utilizamos la Matriz IPM Importancia-Desempeño para determinar las habilidades y competencias requeridas para la gestión de la cadena de suministro

Los resultados muestran que las habilidades de mayor desempeño de las empresas, pero de bajo rendimiento de los estudiantes son: (1) Finanzas y gestión presupuestarias y (2) habilidades de tecnología de la información. Con este déficit recomendamos reforzar estas habilidades a través de nuevas asignaturas, metodologías de la enseñanza innovadoras, prácticas laborales y talleres en empresas para mejorar el desempeño de los futuros profesionales.

\section{ANTECEDENTES}

El concepto de la cadena de suministro ha evolucionado en el sentido que integra como criterio fundamental a las personas para el éxito. Para que la empresa logre los objetivos, requiere que su mano de obra desarrolle las habilidades y competencias necesarias y para lograrlo las universidades deben establecer una enseñanza teórica y práctica que infunda el conocimiento de conceptos aplicables en el campo laboral. A través del estudio realizado por Rahman y Qing (2014) se reconoció un grupo de habilidades y competencias necesarias en la cadena de suministro. Autores como Mangan y Christopher (2005), Gammelgaard y Larson (2001) llevaron a cabo una revisión de literatura, la cual les permitió identificar las principales habilidades estudiadas. A partir de lo anterior, se presentan las 41 habilidades y competencias necesarias en el profesional orientado a la cadena de suministro: Aplazamiento/ reprogramación, benchmarking, cadena de suministro inversa, capacidad para escuchar, capacidad para tomar decisiones, capacidad para ver el 'panorama general', capacidad para planificar, capacidad para priorizar, capacidad para trabajar en equipo, conocimiento de las últimas tecnologías, conocimiento de software, conocimiento de soporte de piezas, capacidad para solucionar problemas, compras, conceptos de cadena de suministro, conocimiento de problemas ambientales de la SC, conocimiento de costos de la cadena de suministro, conocimiento de la cultura organizacional, conocimiento de la industria, conocimiento de las diferencias culturales, conocimiento de las diferencias infraestructurales, finanzas y gestión presupuestaria, flexibilidad, gestión de almacenes, conocimiento multifuncional, conocimientos de subcontratación, conocimientos funcionales, creatividad, EDI / codificación de barras, eliminación de desechos, ética en los negocios, gestión de transporte, habilidad "justo a tiempo" (JIT), habilidad analítica, habilidad con el uso del computador, habilidades de hoja de cálculo, habilidad de coordinación interfuncional, habilidad de hacer contactos (networking), gestión de inventario, gestión de la calidad, gestión de la diversidad, gestión de proyectos, gestión de relaciones con clientes, gestión del tiempo, habilidad de negociación, habilidades cuantitativas y estadísticas, habilidades de bases de datos, habilidades de comunicación escrita y oral, habilidades tecnológicas, habilidades interpersonales, integración de flujos de información - interna y externa, liderazgo, manejo de conflictos, manejo de equipo de oficina, manejo de las TIC's, manejo de materiales, manejo de mercancía devuelta, mejora continua, mejora de procesos de negocio, mentalidad global, motivación, normas ISO 14000, orientación hacia el trabajo en equipo, planificación de los requisitos de distribución, previsión de la demanda, procesamiento de órdenes, razonamiento crítico, regulación del transporte (conocimientos), responsabilidad social y ubicación de instalaciones.

Además, se le adicionaron siete habilidades identificadas en el análisis bibliométrico del estudio Gómez-Cedeño M. et al. (2017), relacionada a habilidades y competencias necesarias en el éxito de la cadena de suministro. Gómez-Cedeño M. et al. (2017) establecen que: (1) mentalidad global, (2) flexibilidad, (3) responsabilidad social, (4) finanzas y gestión presupuestaria, (5) gestión de relaciones con clientes, (6) mejora continua y (7) manejo de la diversidad [1].

Con estas aportaciones se identificaron un total de 48 habilidades y competencias para la SC, que son de importancia para identificar las debilidades de nuestros estudiantes universitarios en el área de Panamá Oeste y de esta manera las instituciones educativas públicas o privadas ejerzan cambios en los planes de estudio, fortaleciendo de esta manera a los futuros profesionales que les proporcionarán el éxito a las empresas.

\section{METODOLOGÍA}

Para determinar las habilidades y competencias que deben aplicar los futuros profesionales, se realizó una investigación descriptiva, cuya finalidad es de observar, describir y analizar las habilidades necesarias hacia la cadena de suministro.

Del estudio Gómez-Cedeño M. et al. (2017) que establece un conjunto de 48 habilidades y competencias necesarias para el éxito de los profesionales de la cadena de suministro del cual 41 de las habilidades identificadas fueron extraídas del estudio de Rahman y Qing (2014) y los siete restantes de una revisión literaria en temas relacionados a cadena de suministro. 
El modelo Gómez-Cedeño M. et al. (2017), propone agrupar las habilidades y competencias en cuatro conjuntos desarrollados por Rahman y Qing (2014): (1) habilidades de gestión general de la cadena de suministro, (2) habilidades de análisis, (3) habilidades de tecnología de la información y (4) habilidades relacionadas con el medio ambiente [3].

Con estas habilidades procedimos a plantear las siguientes preguntas: Identificar, ¿Cuáles son las habilidades y competencias de mayor importancia y las que se desempeñan en la cadena de suministro? y ¿Los estudiantes cumplen con las habilidades para la gestión de la cadena de suministro?

Para la recolección de información, calculamos el tamaño de la muestra mediante la cantidad total de los estudiantes encuestados proporcionando un total de 60 encuestas que debemos realizar, utilizando un margen de error del $8 \%$ y un nivel de confianza del $95 \%$. El formato de la encuesta constaba de las 48 habilidades y competencias para la SCM, en el cual consta de dos columnas de importancia y la otra de desempeño evaluando de este modo, a los estudiantes de la Licenciatura de Ingeniería Industrial y Logística y Transporte Multimodal de 3er y 4to año, de turno matutino y vespertino, en la Universidad Tecnológica de Panamá Centro Regional Panamá Oeste y a los gerentes de algunas empresas de Cadena de Suministro en la Provincia de Panamá Oeste.

Con los resultados obtenidos se procedió al análisis de los datos utilizando la matriz de Importancia desempeño (IPM) mediante el uso del software de EXCEL. La matriz IPM permite ubicar en el eje horizontal la importancia percibida de las características de baja a alta, y en el eje vertical el rendimiento percibido de las características de baja a alta, así formando los siguientes cuadrantes: (1) baja prioridad, (2) posible exceso, (3) concentrarse aquí y (4) mantener el buen trabajo. Evaluando de este modo el IPM en el IV cuadrante de "concentrarse aquî", percepción alto-bajo, donde este cuadrante plasmará las habilidades que requieren alta importancia, pero el desempeño de estas es bajo, esto quiere decir que la institución, en este caso la UTP Panamá Oeste, deberá prestar atención.

\section{RESULTADOS}

Las cinco principales habilidades importantes según los estudiantes UTP - Panamá Oeste

En la figura 1, nos muestra las cinco habilidades más importantes según los estudiantes encuestados del centro, las cuales son: el conocimiento de las últimas tecnologías, gestión de la calidad, gestión de relaciones con clientes, mejora continua, y gestión de inventario.

Las cinco principales habilidades que desempeñan los estudiantes UTP- Panamá Oeste

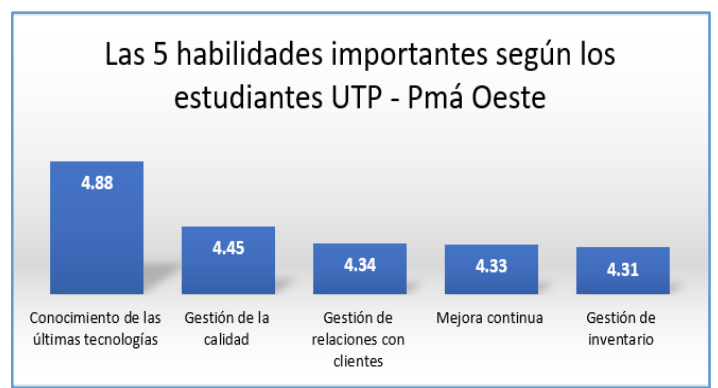

Figura 1. Principales habilidades importantes según los estudiantes - Pmá Oeste.

La figura 2, da a conocer en orden descendente las principales habilidades que desempeñan los estudiantes, las cuales contaron con mayor votación: orientación hacia el trabajo en grupo, habilidades interpersonales, habilidades de comunicación oral, habilidades de comunicación escrita, y responsabilidad social.

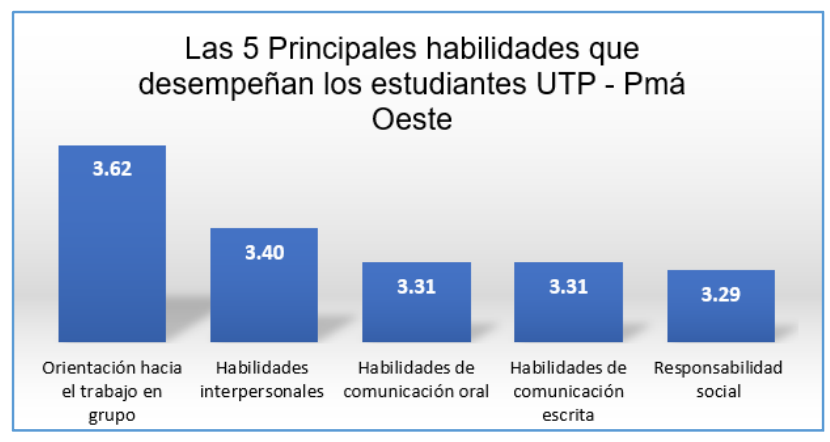

Figura 2. Principales habilidades importantes según los profesionales Panamá Oeste.

Mediante un análisis de la Matriz importancia y desempeño (IPM) de los Estudiantes UTP - Panamá Oeste

Mediante un análisis al (IPM), se muestra la matriz sobre la importancia y desempeño según los estudiantes encuestados del C.R.P.O.

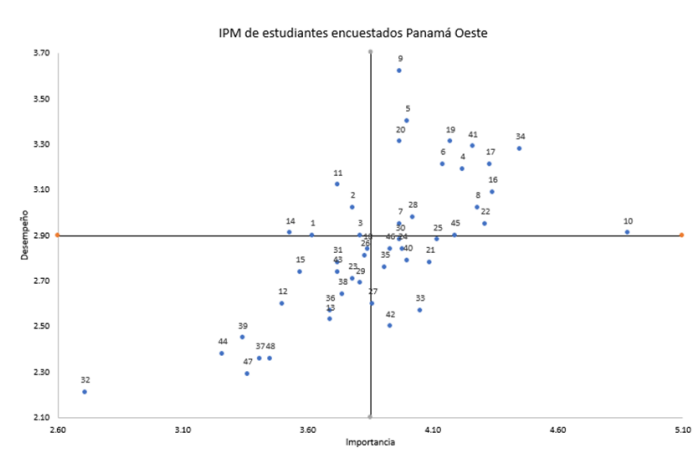

Figura 3. Matriz importancia y desempeño de los Estudiantes UTP - Panamá Oeste. 
Luego de la realización del gráfico, para la IPM según los estudiantes podemos destacar que hay habilidades que son importantes pero su desempeño no cumple con los estándares requerido en las empresas por lo que sugerimos se tomen medidas al respecto para mejorar la situación de las siguientes habilidades: (1) Gestión de transporte, (2) Gestión de almacenes , (3) Planificación de la distribución, (4) Regulación del transporte, (5) Conocimiento de costos de la cadena de suministro , (6) Finanzas y gestión presupuestaria , (7) Conocimiento de problemas ambientales de la Cadena de Suministro, (8) Conocimiento de software , (9) Habilidad de análisis estadísticos y (10) Habilidad de tecnología de la información.

Las cinco principales habilidades importantes según los profesionales - Panamá Oeste.

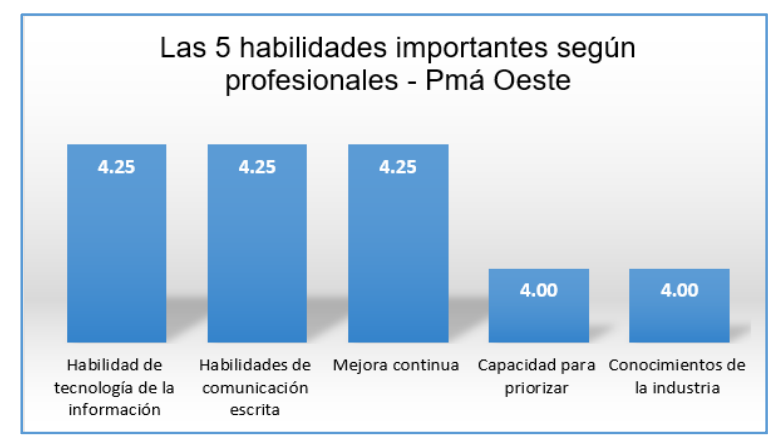

Figura 4. Principales habilidades importantes según profesionales-Panamá Oeste

En la figura\# 4, se observa de acuerdo con el promedio en orden descendente las principales habilidades importantes según los profesionales, son: habilidad de tecnología de la información, habilidades de comunicación escrita, mejora continua, capacidad para priorizar, y conocimientos de la industria, como las más destacadas.

\section{Las cinco principales Habilidades importantes según los profesionales - Panamá Oeste}

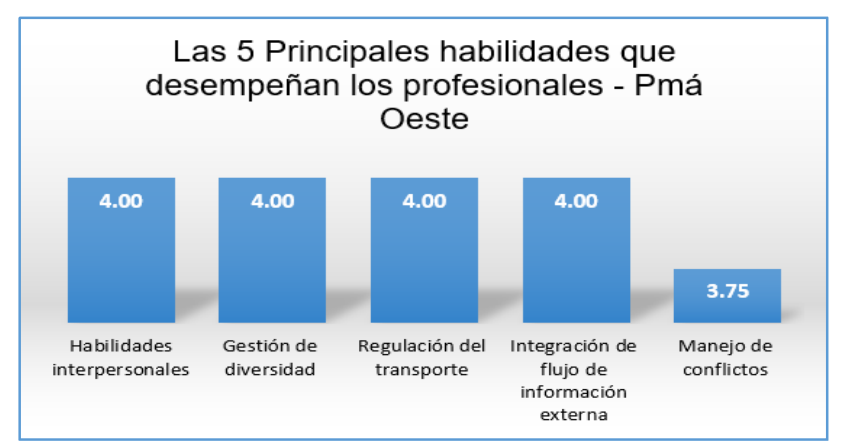

Figura 5. Principales habilidades que desempeñan los profesionales Panamá Oeste
En la figura 5, podremos observar las habilidades con mayor desempeño según los profesionales encuestados en Panamá Oeste, por lo cual las habilidades interpersonales, gestión de diversidad, regulación del transporte, integración de flujo de información externa y manejo de conflictos cuentan con mayor votación.

Matriz importancia y desempeño de los profesionales Panamá Oeste.

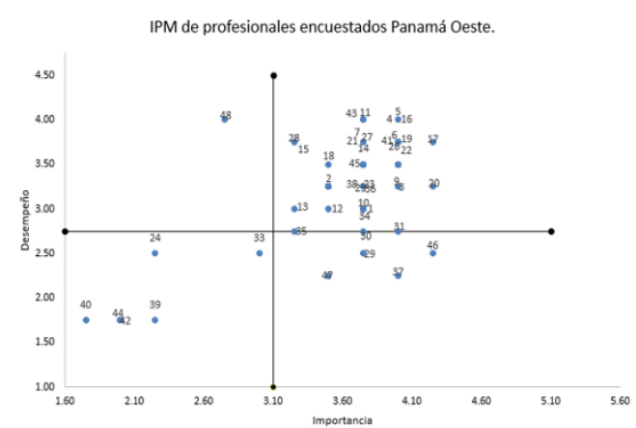

Figura 6. Matriz importancia y desempeño de los Profesionales- Panamá Oeste.

En la siguiente gráfica para la IPM según los profesionales, se destaca que hay habilidades que son importantes, pero su desempeño no es el adecuado, por lo que sugerimos se tomen medidas al respecto para mejorar la situación en las siguientes habilidades: (1) Ubicación de las instalaciones, (2) Manejo de materiales, (3) Gestión de Proyectos, (4) Finanzas y gestión presupuestaria, (5) Normas ISO 14000 , (6) Habilidad de tecnología de la información, (7) Integración de flujo de información interna.

\section{Comparaciones de IPM de estudiantes y profesionales -} Panamá Oeste.

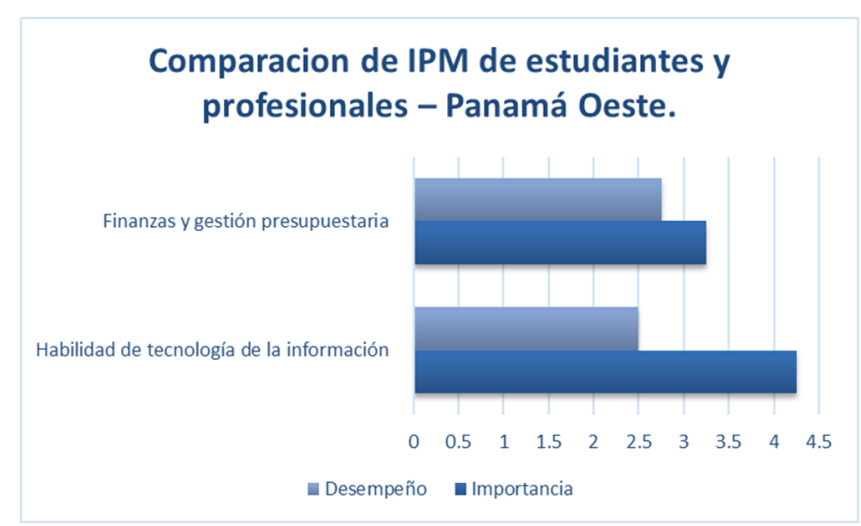

Figura 7: Comparaciones de IPM de estudiantes y profesionales - Panamá Oeste.

En la figura 7, se muestra la comparación por separado a los estudiantes y profesionales, según los datos tienen dos 
habilidades que son importantes, pero no se desempeñan y estas son: (1) Finanzas y Gestión presupuestarias, (2) Habilidades de tecnología de la Información.

\section{CONCLUSIONES}

Con los resultados obtenidos se pudo concluir que dentro de las cinco habilidades que desempeñan los estudiantes UTPPanamá Oeste, cuatro de ellas pertenecen a las habilidades de análisis de la cadena de suministro y la de menos puntuación pertenece a las habilidades de cadena de suministro, relacionada con el medio ambiente. En cambio, para los profesionales, tres de las que más se desempeñan pertenecen a las habilidades de análisis de la cadena de suministro, una de ellas a las habilidades de tecnología de la información de la cadena de suministro y otra habilidad de la gestión de la cadena de suministro.

Los estudiantes encuestados juntos con los profesionales, ambos destacan que la "mejora continua" es una habilidad de gran importancia para la formación de los futuros profesionales.

Según la matriz IPM los estudiantes UTP - Panamá Oeste cuentan con 10 habilidades que son importantes y que no desempeñan, de las cuales la "habilidad de tecnología de la información" es de gran importancia para los profesionales.

Y, por último, los estudiantes encuestados desempeñan la "habilidad de relaciones interpersonales" y "habilidades de comunicación escrita" que son de gran importancia para los profesionales.

\section{RECOMENDACIONES}

Con los resultados obtenidos, podemos recomendar a la Universidad Tecnológica de Panamá, sede Panamá Oeste, que genere actividades para promover la habilidad y competencia del análisis de la cadena de suministro, de tecnología de información de la cadena de suministro y la gestión de la cadena de suministro, por lo que es necesario que se implementen como nuevas asignaturas o cursos asociados a la formación a nuestros futuros profesionales. Es importante, además, variar la metodología de la enseñanza en nuestras universidades de Panamá Oeste, en el aspecto que conste de prácticas laborales dentro del plan de estudio y talleres en las empresas, para así implementar el conocimiento que les será útil para el éxito de las empresas. En donde la empresa gane al capacitar y los estudiantes logren tener esa experiencia que dentro de la universidad no se logra transmitir.

\section{RECONOCIMIENTOS}

Primero ante todo queremos agradecer a dios, por ayudarnos a culminar con esta investigación con bien proporcionándonos el cuidado de cada uno en cada trayectoria que hemos realizado; Segundo a nuestras familias por brindarnos el apoyo motivacional; Tercero, a la doctora Milena Gómez-Cedeño por ser nuestra asesora y apoyarnos con todas las herramientas necesarias para realizar este estudio, además de su conocimiento, experiencia, darnos la oportunidad de realizar un seguimiento a su estudio y aprender conocimientos investigativos y estadísticos; cuarto, al Profesor Mario Hernández que nos apoyó para la selección del proyecto a desarrollar y proporcionarnos el contacto de la doctora Milena Gómez y por último al SCI, por facilitarnos el uso de Software del cual nos fue de mucha ayuda para obtener los resultados inmediatos en esta investigación.

\section{REFERENCIAS}

[1] G. G. H. E. Crystal Diane Bramwell Layne, "Habilidades y competencias para los profesionales de la cadena de suministro: Un análisis y revisión curricular en la Universidad Tecnológica de Panamá.” 2017.

[2] M. Gómez-Cedeño and J. M. a Castán, "La importancia de los recursos humanos en la cadena de suministro The importance of human resources in the supply chain," Dir. y Organ., vol. 54, pp. 13-25, 2014.

[3] N. E. Flores, "Análisis Bibliométrico Sobre La Formación Del Recurso Humano En La Gestión De La Cadena De Suministro," p. 143Con el pasar de los años se ha dado m, 2017. 